\title{
SIMULAÇÃO DOS EFEITOS CONCORRENCIAIS DA FUSÃO COPENE-BRASKEM
}

\author{
Arthur Barrionuevo Filho ${ }^{\S}$ \\ Cláudio Ribeiro Lucinda ${ }^{\text {a }}$
}

\begin{abstract}
RESUMO
Neste artigo são simuladas as implicações concorrenciais decorrentes da compra do controle da empresa COPENE pelo grupo BRASKEM. Mais especificamente, tal operação diz respeito aos efeitos da concentração horizontal no mercado de polietilenos (PEAD, PEBD e PEBDL), e da integração vertical no mercado de eteno. Para avaliar os efeitos da operação, foi realizada uma análise que simulou o comportamento das empresas produtoras de polietilenos e eteno em termos de fixação de preços e quantidades ofertadas. As principais conclusões destas simulações, partindo da formação da empresa BRASKEM, são: (i) elevação do preço médio dos polietilenos no mercado brasileiro; (ii) redução da quantidade total de polietilenos consumida; (iii) elevação da concentração no setor, medida pelo Índice Herfindahl-Hirschmann; (iv) maior participação de mercado da empresa BRASKEM, o que reforça sua posição dominante.
\end{abstract}

Palavras-chave: concentração horizontal, integração vertical, defesa da concorrência, indústria química, polietileno.

\section{ABSTRACT}

In this paper, we aim to analyze the consequences for the competition as regards the purchase of control of COPENE enterprise by the BRASKEM group. Specifically, such operation increases the degree of horizontal concentration in Brazilian Polyethylene market (PEAD, PEBD, PEBDL), as well as the concentration in the market for ethene. In order to evaluate the consequences of these operations, an analysis was carried out in order to simulate the behavior of producing companies of polyethylene and ethene as regards prices and quantities. The main conclusions of these simulations, from the formation of BRASKEM enterprise: (i) increase in average prices of polyethylene in Brazilian Market; (ii) decrease in the total quantity of polyethylene consumed; (iii) Increase in the Herfindahl-Hirschman Index in the sector; (iv) greater market share of BRASKEM, reinforcing its dominant position.

Key words: horizontal concentration, vertical integration, antitrust, chemical industry, polyethylene.

JEL classification: L11, L13.

§ Fundação Getúlio Vargas - Escola de Economia de São Paulo. E-mail: abarrio@fgvsp.br.

a Fundação Getúlio Vargas - Escola de Economia de São Paulo. E-mail: claudiolucinda@fgvsp.br.

Endereço para contato: R. Itapeva, 474, $11^{\circ}$ andar - Bela Vista - São Paulo - SP - CEP 01420-001. Fone: (11) 3281-7765.

Recebido em junho de 2005. Aprovado em novembro de 2006. 


\section{INTRODUÇÃO}

O controle acionário da COPENE, empresa produtora de eteno do Pólo de Camaçari, Bahia, pela empresa Odebrecht Química, coloca duas questões concorrenciais importantes. ${ }^{1}$ A primeira, mais imediata, diz respeito à concentração horizontal no mercado de polietileno $\left(\mathrm{PEAD}^{2}, \mathrm{PEBD}^{3} \mathrm{e}\right.$ $\mathrm{PEBDL}^{4}$ ) envolvendo a empresa POLIALDEN e o Grupo Odebrecht. A segunda, a concentração na produção brasileira de eteno, insumo essencial para a produção dos polietilenos.

O Grupo Odebrecht, após esta operação, passou a participar do controle dos dois principais pólos produtores de eteno no Brasil: Camaçari, Bahia e Rio Grande do Sul. Como se sabe, o eteno é um produto cujo transporte exige condições especiais (é um gás que se liquefaz apenas a temperaturas muito reduzidas) e tem custos elevados. O fornecimento de eteno para uma planta de polietileno, a partir de outro pólo petroquímico, torna inviável economicamente a planta de polietileno, pois a participação da matéria-prima eteno é muito significativa no custo do mesmo. Além disto, o abastecimento da mesma a partir de outro pólo petroquímico elevaria seus custos de tal maneira que tornaria impossível a fixação de preços competitivos em relação a concorrentes com plantas próximas ao produtor.

Deste modo, abre-se, hipoteticamente, a possibilidade de manipulação de preços ou quantidades ofertadas de eteno, para elevar preços e lucros no mercado de polietilenos. Isto acarretaria uma redução do bem-estar dos consumidores, um aumento de lucros da empresa integrada verticalmente e redução de participação de mercado dos concorrentes.

Para a investigação desta hipótese devemos começar analisando a estrutura do setor. Com este fim, e considerando que inexiste a diferenciação de produtos no caso dos polietilenos, o modelo mais adequado segundo a literatura é o de Cournot. ${ }^{5}$ Este modelo considera que a variável competitiva chave em oligopólios é a capacidade de oferta de curto prazo de cada firma que definirá sua estratégia de preços e quantidades ofertadas. ${ }^{6}$ As principais características desse modelo são: a) existem barreiras à entrada, no caso o tamanho do investimento em relação ao mercado e o tempo necessário para colocá-lo em operação; b) o produto é não diferenciado; e c) a capacidade produtiva apresenta indivisibilidades técnicas, portanto, evolui por patamares e não incrementalmente. Neste modelo, a teoria econômica estabelece uma relação entre os preços e margens de lucros de uma indústria como resultado do seu grau de concentração, mensurado pelo Índice Herfindahl-Hirschmann (HHI).

O uso do modelo de Cournot para mensurar os efeitos de concentrações horizontais foi aperfeiçoado por Farrell e Shapiro (1990). Estes autores avaliam os efeitos de uma concentração horizontal e como a autoridade antitruste deve responder a ela. A principal conclusão é que uma fusão entre duas empresas no mesmo setor pode gerar eficiências que podem contrabalançar os efeitos sobre os preços ${ }^{7}$ da concentração. Para eles as fusões podem ter três tipos de efeitos: a) nenhum impacto sobre os custos marginais, provocando aumento de preços; b) realocação da produção para

1 A aquisição do controle acionário da COPENE por parte da Odebrecht Química ocorreu dia 25 de junho de 2001 em leilão realizado pelo Banco Central do Brasil.

2 Polietileno de Alta Densidade.

3 Polietileno de Baixa Densidade.

4 Polietileno de Baixa Densidade Linear.

5 Uma introdução sobre o tema pode ser encontrada em Tirole (1989, cap. 5).

6 Conforme um trabalho clássico de Kreps e Scheinkman (1983), que mostram que o resultado tradicional de Cournot pode ser verificado como o equilíbrio de um jogo em dois estágios; no primeiro ocorre a decisão sobre a capacidade e no segundo as empresas competem por preços.

7 A integração horizontal pode induzir uma realocação da produção, das plantas menos eficientes para as mais eficientes e pode também gerar sinergias, de escala ou aprendizado, que aumentam a eficiência produtiva após a fusão. 
as plantas mais eficientes, gerando um aumento de eficiência limitado e somente no curto prazo; c) criação de sinergias, como economias de escala ou de aprendizado.

Assim, não necessariamente um aumento do HHI provocará somente aumento de preços. Todavia, estes ganhos não estão necessariamente presentes e sua magnitude deve ser suficientemente elevada para compensar os impactos da concentração. No caso presente, como a empresa BRASKEM já operava plantas de polietilenos e dado o grau de utilização pré-fusão das plantas, ${ }^{8}$ dificilmente se pode esperar algum ganho como os descritos em (b) e (c) acima, ainda que para alguns autores como Santana et al. (2003) existia à época uma grande possibilidade de ocorrência de ganhos por meio de sinergias e realocação da produção para plantas mais eficientes. ${ }^{9}$ Desta forma, uma análise mais cuidadosa do mercado envolvido se faz necessária para mensurar os efeitos em termos de bem-estar dos consumidores.

O segundo ponto, mais duradouro em termos concorrenciais, da concentração gerada pela formação da empresa BRASKEM é o aspecto de integração vertical da operação. A análise de efeitos anticoncorrenciais de integrações verticais, depois de ter sido praticamente descartada, no período de predomínio da Escola de Chicago, ${ }^{10}$ foi retomada sobre novas bases, com maior fundamentação econômica.

Ordover, Saloner e Salop (1990) discutem os motivos do abandono da teoria clássica dos efeitos anticompetitivos das integrações verticais e quais os fundamentos para retomá-la. Um dos principais motivos apontados pela Escola de Chicago para o abandono da análise de efeitos anticoncorrenciais de integrações verticais é que, em havendo uma integração vertical, não faria sentido para a empresa integrada reduzir suas vendas de insumos para os concorrentes, pois isso reduziria seus lucros. Contudo, estes autores desenvolvem um novo modelo, mostrando que sob certas condições pode ser lucrativo reduzir a venda de insumos para os concorrentes, aumentando os preços dos insumos e o custo dos concorrentes, repassando este maior custo para o preço do produto final. A empresa integrada acompanharia o preço dos concorrentes e aumentaria seu lucro no mercado de produto final, compensando a redução de vendas no mercado de insumos.

No caso em análise, a situação seria ainda mais favorável a uma conduta para aumentar os preços por parte da empresa integrada, pois a empresa BRASKEM tem uma planta de polietileno no pólo da Bahia, portanto, nem seria necessário reduzir as suas vendas de eteno.

A análise realizada a seguir considerará também os efeitos advindos dos aspectos de integração vertical sobre o mercado de polietileno no Brasil. Para tanto, devem ser mensurados os impactos nos preços e quantidades de equilíbrio no mercado de polietileno, a partir da capacidade que a empresa BRASKEM terá, nos próximos anos, de fixar preços e quantidades de eteno supridas aos seus competidores e, deste modo, influenciar preços e quantidades de equilíbrio no mercado de polietileno.

Para tanto, é necessário inicialmente construir um modelo sobre o funcionamento do mercado. Na próxima seção será realizada a análise econométrica, para estimar parâmetros e relações que descrevem o funcionamento do setor - em especial, estimativas de Custo Marginal e funções de Demanda pelos diferentes produtos.

8 Segundo ABIQUIM (2005), a BRASKEM possuía uma capacidade instalada de produção de PEAD de 330 mil toneladas/ano, de PEBD igual a 210 mil toneladas/ano e de 300 mil toneladas/ano de PEBDL.

9 Segundo Santana et al. (2003, p. 172): "A estratégia de integração de ativos permitiu assim a identificação de sinergias que beneficiam toda a cadeia produtiva[...] A nova perspectiva em termos de negócios químicos e petroquímicos dessa megaempresa evidencia-se em função do perfil da mesma, que é similar aos modelos mundiais predominantes nesse setor, em que as empresas atuam concentradas, integradas e com escala de produção."

10 Recomenda-se Riordan e Salop (1995) para uma análise do tema. 
$\mathrm{Na}$ seção três serão realizadas as simulações, a partir dos custos e demandas estimados, para verificar os resultados sobre o mercado de polietileno. A seção quatro conclui acerca dos resultados concorrenciais e sobre o bem-estar dos consumidores no mercado de polietileno.

\section{MODELAGEM DO SETOR: CUSTOS E DEMANDA}

O primeiro passo da análise envolve a construção de um modelo de competição imperfeita que replica as características do setor de polietilenos (PEAD, PEBDL e PEBD) no Brasil. A partir deste modelo, extraem-se conclusões sobre os possíveis efeitos decorrentes da fusão em análise.

O fundamento teórico para as estimativas é o modelo de Cournot com diferentes custos marginais que originam diferentes participações de mercado para cada empresa. Para que este modelo possa ser bem-sucedido, são necessários os seguintes dados: ${ }^{11}$

- Funções de Demanda por segmento e para o mercado como um todo;

- Custos Marginais do setor e por segmento;

- Participações de Mercado.

As participações de mercado utilizadas são baseadas em ABIQUIM (2002). Os outros elementos são de obtenção mais complexa e serão tratados nas seções seguintes.

\subsection{Estimativas de demanda}

O primeiro passo necessário é desenvolver um modelo econométrico para a estimação de valores para as funções demanda relevantes para a análise de mercado. Esta análise econométrica baseia-se nas contribuições de Kadiyali (1996) e Roberts e Samuelson (1988). Este modelo se baseia na estimação - por mínimos quadrados a três estágios (MQ3E ${ }^{12}$ ) - de uma função demanda pelos dois dos principais produtos do setor de polietilenos - PEAD e PEBDL. ${ }^{13}$ Optou-se por esta forma de estimação, envolvendo somente dois produtos, uma vez que do ponto de vista da demanda o PEBDL e o PEBD se mostram bastante similares.

Inicialmente, especificam-se os elementos básicos do modelo para, em um momento subseqüente, derivar a especificação econométrica propriamente dita.

\subsubsection{Demanda por polietileno de alta densidade}

A função demanda por Polietileno de Alta Densidade é dada por:

$$
\mathrm{Q}_{\mathrm{PEAD}}=\beta_{0}+\beta_{1} \mathrm{P}_{\mathrm{PEAD}}+\beta_{2} \mathrm{Q}_{\mathrm{PERF}}+\beta_{3} \mathrm{Q}_{\mathrm{CDI}}+\beta_{4} \mathrm{D} 1
$$

Em que:

- $\mathrm{Q}_{\mathrm{PEAD}}$ - Quantidade Produzida de Polietileno de Alta Densidade;

- $\mathrm{P}_{\mathrm{PEAD}}$ - Preço do Polietileno de Alta Densidade;

- $\mathrm{Q}_{\mathrm{PERF}}$ - Número-Índice representativo da atividade no setor de perfumaria;

- $\mathrm{Q}_{\mathrm{CDI}}$ - Número-Índice representativo da Taxa de Juros de Curto Prazo-CDI;

11 Seguimos, neste particular, a análise de Farrell e Shapiro (1990) supramencionada.

12 Recomenda-se Greene (1996) para uma análise mais aprofundada do tema.

13 Optou-se por estimar demandas separadas somente para o PEAD e para o PEBDL. 
- D1 - Variável Dummy referente ao quarto trimestre de cada ano.

\subsubsection{Demanda por polietileno de baixa densidade linear}

A função demanda por Polietileno de Baixa Densidade Linear é dada por:

$$
\mathrm{Q}_{\mathrm{PEBDL}}=\beta_{5}+\beta_{6} \mathrm{P}_{\mathrm{PEBDL}}+\beta_{7} \mathrm{Q}_{\mathrm{CDI}}
$$

Em que:

- $\mathrm{Q}_{\mathrm{PEBDL}}$ - Quantidade Produzida de Polietileno de Baixa Densidade Linear;

- $\mathrm{P}_{\mathrm{PEBDL}}$ - Preço do Polietileno de Baixa Densidade Linear;

- $\mathrm{Q}_{\mathrm{CDI}}$ - Número-Índice representativo da Taxa de Juros de Curto Prazo-CDI.

Não há variáveis dummy nesta equação, pois elas se mostraram não-significantes.

\subsubsection{Custo marginal do produto}

O custo marginal de produção do produto i (i=PEAD, PEBDL) é igual a:

$$
\mathrm{CMg}_{\mathrm{i}}=\gamma_{0}+\gamma_{1} \mathrm{~L}_{\mathrm{i}}+\gamma_{2} \mathrm{P}_{\text {ETENO }}
$$

Em que:

- $\mathrm{L}_{\mathrm{i}}$ - Remuneração da Mão-de-Obra no setor - Índice IBGE (Pesquisa Mensal do Emprego $-\mathrm{PME})$;

- $\mathrm{P}_{\text {ETENo }}$ - Preço do Eteno.

A partir destas funções, podemos determinar qual será a especificação do modelo econométrico de análise.

\subsubsection{Especificação do modelo econométrico}

Para obter as especificações a serem estimadas, parte-se da premissa que o setor como um todo maximiza a seguinte função de lucros. Observe-se também que, como muitas plantas "reversíveis" podem produzir de maneira alternativa PEAD e PEBDL, a soma das duas equações reflete esta condição tecnológica presente na indústria:

$$
\Pi=\mathrm{P}_{\text {PEAD }} \times \mathrm{Q}_{\mathrm{PEAD}}+\mathrm{P}_{\mathrm{PEBDL}} \times \mathrm{Q}_{\mathrm{PEBDL}}-\mathrm{CMg}_{\mathrm{PEAD}} \times \mathrm{Q}_{\mathrm{PEAD}}-\mathrm{CMg}_{\mathrm{PEBDL}} \times \mathrm{Q}_{\mathrm{PEBDL}}
$$

Esta função lucro, por sua vez, tem duas variáveis (variáveis que as empresas manipulam para maximizar o seu lucro) sobre as quais se realiza a estimação - os preços dos dois produtos. ${ }^{14}$ Substituindo as equações 1 e 2 na função $\Pi$, temos as seguintes condições de primeira ordem - que representariam o lado da oferta do modelo:

$$
\begin{gathered}
\mathrm{Q}_{\mathrm{PEAD}}+\left(\mathrm{P}_{\mathrm{PEAD}}-\mathrm{CMg}_{\mathrm{PEAD}}\right) \beta_{1}=0 \\
\mathrm{Q}_{\mathrm{PEBDL}}+\left(\mathrm{P}_{\mathrm{PEBDL}}-\mathrm{CMg}_{\mathrm{PEBDL}}\right) \beta_{6}=0
\end{gathered}
$$

14 É importante notar que este procedimento tem por objetivo simplesmente derivar uma especificação testável para os parâmetros da demanda por Polietilenos, o que será importante para a análise subseqüente. Na prática, existem mais de dois produtos envolvidos. 
Substituindo a definição de custo marginal derivada da equação 3, tem-se então um sistema de quatro equações, da seguinte forma:

$$
\begin{aligned}
& \mathrm{Q}_{\mathrm{PEADt}}=\beta_{0}+\beta_{1} \mathrm{P}_{\mathrm{PEADt}}+\beta_{2} \mathrm{Q}_{\mathrm{PERFt}}+\beta_{3} \mathrm{Q}_{\mathrm{CDIt}}+\beta_{4} \mathrm{D} 1_{\mathrm{t}}+\varepsilon_{\mathrm{t}} \\
& \mathrm{Q}_{\mathrm{PEBDL}}=\beta_{5}+\beta_{6} \mathrm{P}_{\mathrm{PEBDL}}+\beta_{7} \mathrm{Q}_{\mathrm{CDIt}}+\varepsilon_{\mathrm{t}} \\
& \mathrm{Q}_{\mathrm{PEADt}}=\beta_{1}\left(\gamma_{0}+\gamma_{1} \mathrm{~L}_{\mathrm{PEADt}}+\gamma_{2} \mathrm{P}_{\mathrm{ETENO}}\right)+\beta_{1} \mathrm{P}_{\mathrm{PEADt}}+\varepsilon_{\mathrm{t}} \\
& \mathrm{Q}_{\text {PEBDLt }}=\beta_{6}\left(\gamma_{0}+\gamma_{3} \mathrm{~L}_{\mathrm{PEADt}}+\gamma_{2} \mathrm{P}_{\mathrm{ETENO}}\right)+\beta_{6} \mathrm{P}_{\mathrm{PEBDLt}}+\varepsilon_{\mathrm{t}}
\end{aligned}
$$

Estas quatro equações podem ser estimadas em conjunto por qualquer técnica de estimação de equações simultâneas. É importante observar que houve uma opção de estimar as duas últimas equações acima somadas, para melhorar os resultados da estimação. ${ }^{15}$ Os resultados são:

$$
\begin{aligned}
& \mathrm{Q}_{\text {PEADt }}=33,18-12,07 \mathrm{P}_{\text {PEADt }}+365,88 \mathrm{Q}_{\text {PERFt }}-6132,51 \mathrm{Q}_{\text {CDIt }}+6028,06 \mathrm{D} 1_{\mathrm{t}} \\
& \mathrm{Q}_{\text {PEBDLt }}=41,14-12,26 \mathrm{P}_{\text {PEBDLt }}-5907,32 \mathrm{Q}_{\mathrm{CDIt}} \\
& \mathrm{Q}_{\text {PEADt }}+\mathrm{Q}_{\text {PEBDLt }}=61.755,73-15,07 \mathrm{~L}_{\mathrm{t}}+30,67 \mathrm{P}_{\text {ETENOt }}+12,07 \mathrm{P}_{\text {PEADt }}+12,26 \mathrm{P}_{\text {PEBDLt }}
\end{aligned}
$$

Em que L denota o custo total da mão-de-obra no setor. Adicionalmente foi realizada uma estimativa, também por Mínimos Quadrados a Três Estágios, de uma função demanda para o setor (total de polietilenos), bem como uma função oferta associada com o processo de maximização da empresa. Os resultados foram os seguintes:

$$
\begin{aligned}
\mathrm{Q}_{\mathrm{Tt}} & =111.741,70-43,95 \mathrm{P}_{\mathrm{POLt}}+503,37 \mathrm{Q}_{\mathrm{INDt}} \\
\mathrm{Q}_{\mathrm{Tt}} & =125.366-5,998 \mathrm{~L}_{\mathrm{t}}+15,579 \mathrm{P}_{\mathrm{ETENOt}}+17,326 \mathrm{P}_{\mathrm{POLt}}
\end{aligned}
$$

Em que $\mathrm{Q}_{\mathrm{T}}$ denota a quantidade total de polietileno produzida, $\mathrm{P}_{\mathrm{POL}}$ o preço médio do polietileno - média simples dos três produtos - PEAD e PEBDL - e $\mathrm{Q}_{\mathrm{IND}}$ o número-índice representativo da produção na indústria. Também foram realizadas estimações utilizando-se especificações isoelásticas; os resultados para todas estas especificações estão expostos nos anexos.

Ainda que tenhamos conseguido a identificação dos parâmetros das funções demanda com esta metodologia, não foi possível obter estimativas confiáveis para o Custo Marginal do setor. A principal razão é o fato que as séries que refletem o custo do capital são pouco significantes na análise econométrica. ${ }^{16}$ Ou seja, uma metodologia alternativa é necessária para obter valores de custos marginais, como se verá no próximo item.

15 Os instrumentos utilizados foram os preços dos dois polietilenos, em nível e defasados em até dois períodos, o preço do eteno, em nível e defasado em até dois períodos, além da variável $\mathrm{Q}_{\mathrm{CDIt}}$ e $\mathrm{Dl} 1_{\mathrm{t}}$.

16 Tentou-se utilizar como proxy do custo de capital a taxa de juros dos Certificados de Depósitos Interbancários; no entanto, os resultados não foram significativos. 


\subsection{Custo marginal - metodologia alternativa de estimação}

O passo seguinte na análise, tendo em vista as limitações da metodologia acima, é buscar outras formas de estimação do Custo Marginal. Para tanto, toma-se como ponto de partida o valor dos custos unitários obtidos para cada uma das famílias de produtos acima mencionados.

Os custos foram fornecidos pela empresa POLITENO, com uma periodicidade anual, englobando custos variáveis, parte dos custos fixos, incluindo as despesas com depreciação. Tendo em vista que estes custos estavam em reais correntes, eles foram deflacionados utilizando as seguintes regras:

- Preço do Eteno: deflacionado de acordo com a série de preços do eteno fornecida pela empresa POLITENO;

- Preço da Energia Elétrica: deflacionado de acordo com o índice de preços da Energia Elétrica;

- Demais Insumos e Custos Fixos: deflacionados de acordo com o IPA-OG (Índice de Preços no Atacado - Oferta Global) para o setor da Indústria Química;

- Custo da Mão-de-Obra: deflacionado de acordo com a evolução do custo da mão-de-obra calculada pela ABIQUIM (Associação Brasileira da Indústria Química - Relatório Custo da Mão-de-Obra).

Aplicando esta regra, são obtidos os seguintes valores médios para o Custo Médio de Produção, ao longo do período entre 1999 e 2002: $\mathrm{R} \$ 771,00$ para o PEAD, R \$ 801,00 para o PEBDL e $\mathrm{R} \$ 732,00$ para o PEBD.

Este deflacionamento fez com que os valores resultantes estejam expressos em Reais de 1998. Para estimar valores para os custos marginais a partir dos custos médios existem duas etapas a serem realizadas. Em primeiro lugar, para a obtenção dos custos médios em dólares, estes valores foram convertidos pelo dólar médio de 1998 e a média destes custos para o período 1999-2002 foi computada para chegar aos custos médios de produção por segmento. Os valores obtidos foram: US\$ 638 para o PEAD, US\$ 662 para o PEBDL e US\$ 605 para o PEBD.

O passo seguinte foi estimar a relação entre custos médios e custos marginais no setor. Para tanto, foi realizada uma análise de fronteira estocástica ${ }^{17}$ para determinar a fronteira eficiente do setor, e a partir daí avaliar como os custos médios evoluem em relação ao valor adicionado na produção.

Estes resultados, expostos no Apêndice, indicam uma elasticidade-produção dos custos da ordem de 0,65 . Ou seja, para um aumento de $1 \%$ no valor adicionado no setor químico, os custos totais se elevam em $0,65 \%$. Lembrando a definição de elasticidade:

$$
\varepsilon_{Y}^{C T}=\frac{\partial C T}{\partial Y} \times \frac{Y}{C T}
$$

Em que:

17 Foram selecionadas 10 empresas do setor químico, com dados trimestrais de 1999/1 a 2003/2. Definiu-se o Valor Adicionado $\left(\mathrm{VA}_{\mathrm{it}}\right)$ em cada empresa para cada um dos trimestres como a diferença entre Receita Bruta e Impostos sobre Vendas e Custos Operacionais. O Custo Total $\left(\mathrm{CT}_{\mathrm{it}}\right)$ é definido como a soma dos Custos Operacionais e Custos dos Produtos Vendidos. A análise de Fronteira Estocástica baseou-se na seguinte especificação:

$\ln \left(\mathrm{CT}_{\mathrm{it}}\right)=\beta_{0}+\beta_{1} \ln \left(\mathrm{VA}_{\mathrm{it}}\right)+\varepsilon_{\mathrm{it}} \mathrm{v}_{\mathrm{it}}$

Os resultados desta análise estão expostos no Apêndice. Recomenda-se Battese e Coelli (1992) para mais detalhes sobre o modelo. 
- Y - Valor da Produção;

- CT - Custo Total da Produção;

- $\quad[(\partial \mathrm{CT}) /(\partial \mathrm{Y})]-$ Derivada Parcial do Custo com relação à Produção.

Desta forma, obtêm-se estimativas para o custo marginal iguais ao custo médio multiplicado por 0,65 . Este valor indica a existência de economias de escalas na indústria química, pois o custo marginal é o custo variável adicional, enquanto o custo médio e o valor adicionado incluem também os custos fixos. Com este valor de 0,65 , os valores para os custos marginais são de US $\$ 416,52$ para o PEAD, US\$ 432,59 para o PEBDL e US\$ 395,24 para o PEBD.

Note-se que qualquer modelo que simular o comportamento de uma indústria deverá trabalhar com algumas simplificações. No caso em questão, considera-se que a relação constante entre custos médios e marginais, levando-se em consideração as economias de escala, é aceitável como uma aproximação linear.

\section{Resultados do MODElo DE ANÁlise}

Partindo das características da indústria, foi utilizado o Modelo de Cournot para a obtenção de estimativas dos efeitos sobre preço, quantidade e concentração de mercado - mensurada pelo Índice de Herfindahl-Hirschman - no setor de polietilenos. No modelo de Cournot as firmas atuantes no mercado escolhem como variável estratégica a quantidade a ser produzida em um dado instante do tempo, considerando as escolhas das outras firmas, gerando assim um equilíbrio de mercado. Para a utilização deste modelo para o caso brasileiro, os seguintes dados são necessários:

1. Custos Marginais das diferentes firmas;

2. Função Demanda do setor.

A função demanda do setor foi obtida no item 2.1 acima, enquanto que a estimativa de Custo Marginal foi obtida no item 2.2. Partindo do Modelo de Cournot aplicado ao setor de polietilenos, o processo de decisão das diferentes empresas considera que as plantas do setor são de capacidade reversível (em uma proporção aproximadamente de 1:1) entre as duas linhas de produtos (PEAD e PEBDL). Isto leva a uma tomada de decisão em que a variável chave passa a ser a quantidade total de polietilenos produzida pelos competidores.

Com esse instrumental de análise, padrão em estudos sobre este tipo de indústria, foram realizadas simulações referentes aos efeitos sobre as variáveis de mercado. Dois tipos de simulação foram usados:

- Participações de Mercado Endógenas: uma vez que os produtos são não diferenciados, as participações de mercado seriam, portanto, derivadas de custos marginais assimétricos. A manipulação de preços do eteno, principal componente do Custo Marginal, ocasiona mudança de participações de mercado e conseqüentemente de grau de concentração e de preços e quantidades de equilíbrio no mercado de polietileno;

- Participações de Mercado Exógenas: as participações de mercado das diferentes firmas são dadas (exógenas) e decorrem do grau de utilização de capacidade e das suas diferentes capacidades produtivas totais. A manipulação da quantidade de eteno fornecida a cada planta ocasiona mudança de participações de mercado e, conseqüentemente, de grau de concentração e de preços e quantidades de equilíbrio no mercado de polietileno. 
Estas duas simulações, usadas alternativamente, permitem testar a "robustez" dos efeitos da integração vertical mencionada, decorrentes do controle sobre o preço e a quantidade de eteno fornecida nos pólos da Bahia e Rio Grande do Sul pelas empresas COPENE e COPESUL.

\subsection{Participações de mercado endógenas}

A simulação que chega às participações de mercado a partir dos custos marginais tem como base os dados citados anteriormente, em que o preço-base da eteno é o da POLITENO. Esta simulação foi realizada em duas versões. Na primeira delas cada linha de produto é analisada isoladamente, desconsiderando a possibilidade de reversão de capacidade entre as distintas linhas de produtos.

\subsubsection{Análise por segmento de mercado}

As simulações partiram da calibragem dos valores de um modelo de Cournot para refletir as participações de mercado por segmento de cada um dos grupos. As variáveis de ajuste do modelo foram os custos marginais dos outros grupos, para os quais não existe uma estimativa confiável e em especial do preço do eteno que responde pela maior parte do custo marginal.

A partir destes valores obtidos, a simulação foi realizada por meio da variação dos Custos Marginais da empresa equivalente à POLITENO no modelo. Os resultados estão expostos nas figuras a seguir. ${ }^{18}$ Para cada um dos produtos faz-se uma estimação da relação entre o valor do custo marginal e os impactos sobre o HHI, o preço de mercado e a quantidade consumida.

\section{Figura 1 - Índice Herfindahl-Hirschman, preço e quantidade - PEBDL}
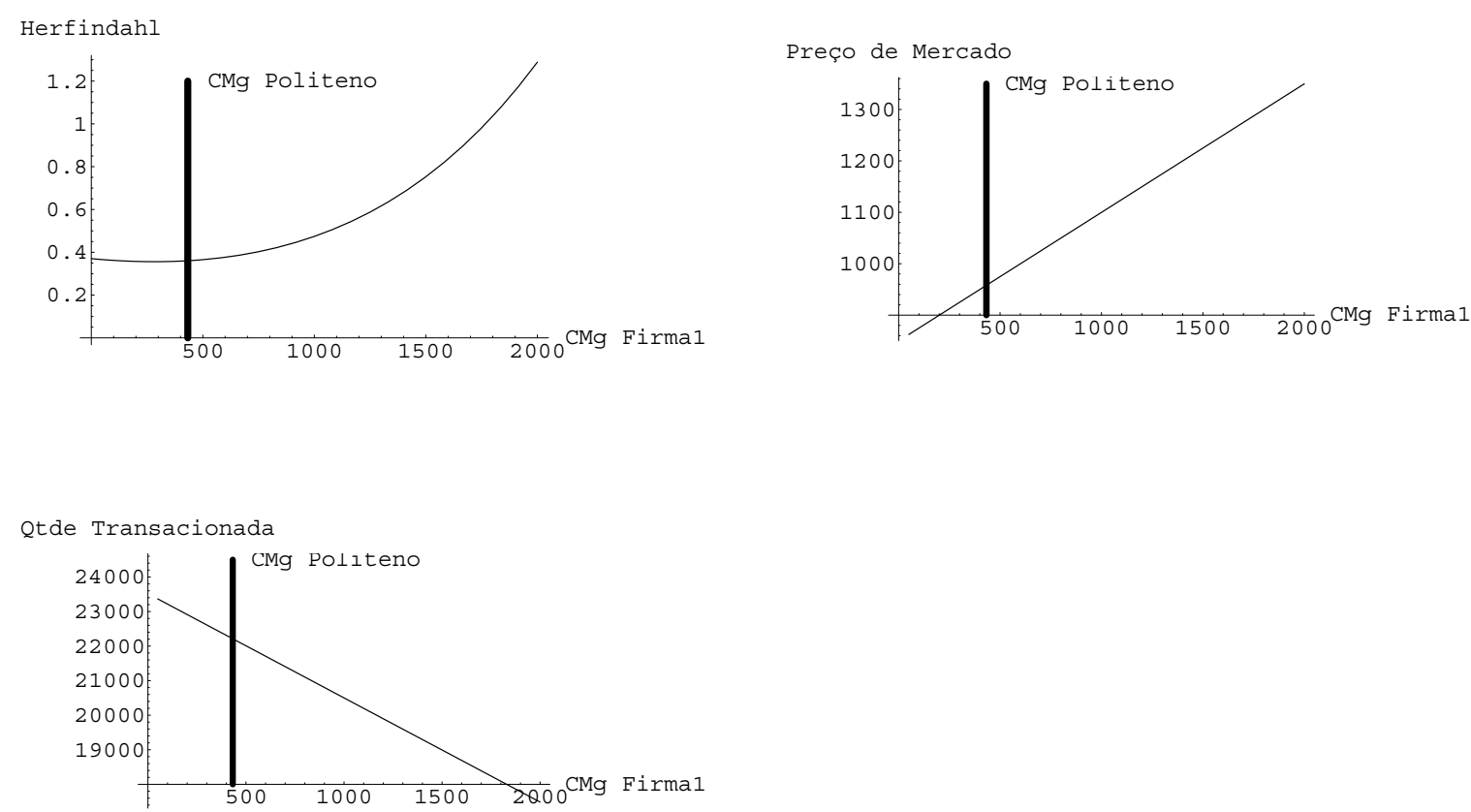

Fonte: Elaboração dos Autores.

18 O Software utilizado foi o Mathematica, versão 4.1. 
Figura 2 - Índice Herfindahl-Hirschman, preço e quantidade - PEAD
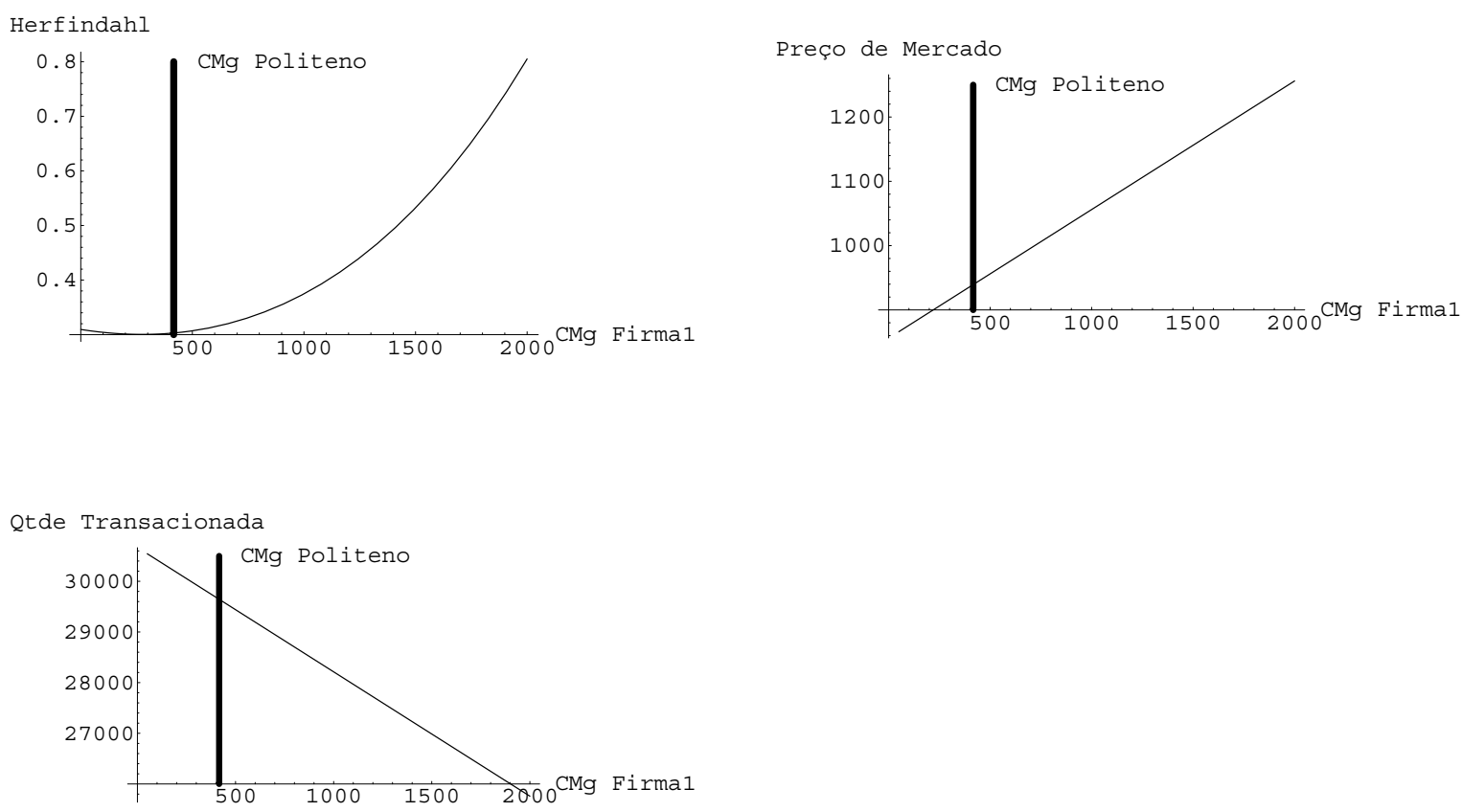

Fonte: Elaboração dos Autores.

As Figuras 1 e 2 mostram que a elevação do custo marginal da empresa POLITENO acima dos valores observados atualmente faz com que: (1) se eleve o grau de concentração na indústria; (2) eleve-se o preço de mercado em cada um dos segmentos e (3) reduza-se a quantidade transacionada em cada um dos tipos de polietileno. Estes resultados foram atingidos considerando o aumento do custo marginal e são consistentes com o aumento de poder de mercado da empresa BRASKEM, tendo em vista o seu controle da produção nacional de eteno e a sua capacidade de aumentar o preço deste insumo.

\subsubsection{Análise do mercado como um todo}

A seguir foi modelado o mercado de polietileno como um todo. Para tanto, foram estimadas as participações de mercado de cada um dos principais grupos, e foram calibrados os custos marginais de cada grupo de forma a refletir estas participações. ${ }^{19}$ Os resultados de simulação similar à realizada acima estão na figura a seguir.

19 A agregação dos diferentes tipos de polietileno foi realizada tendo em vista a conversão de capacidade de produção entre PEAD e PEBDL. Quando se considera a restrição de capacidade, é difícil determinar como alocá-la entre os dois produtos. 
Figura 3 - Índice Herfindahl-Hirschman, preço e quantidade no setor de polietilenos
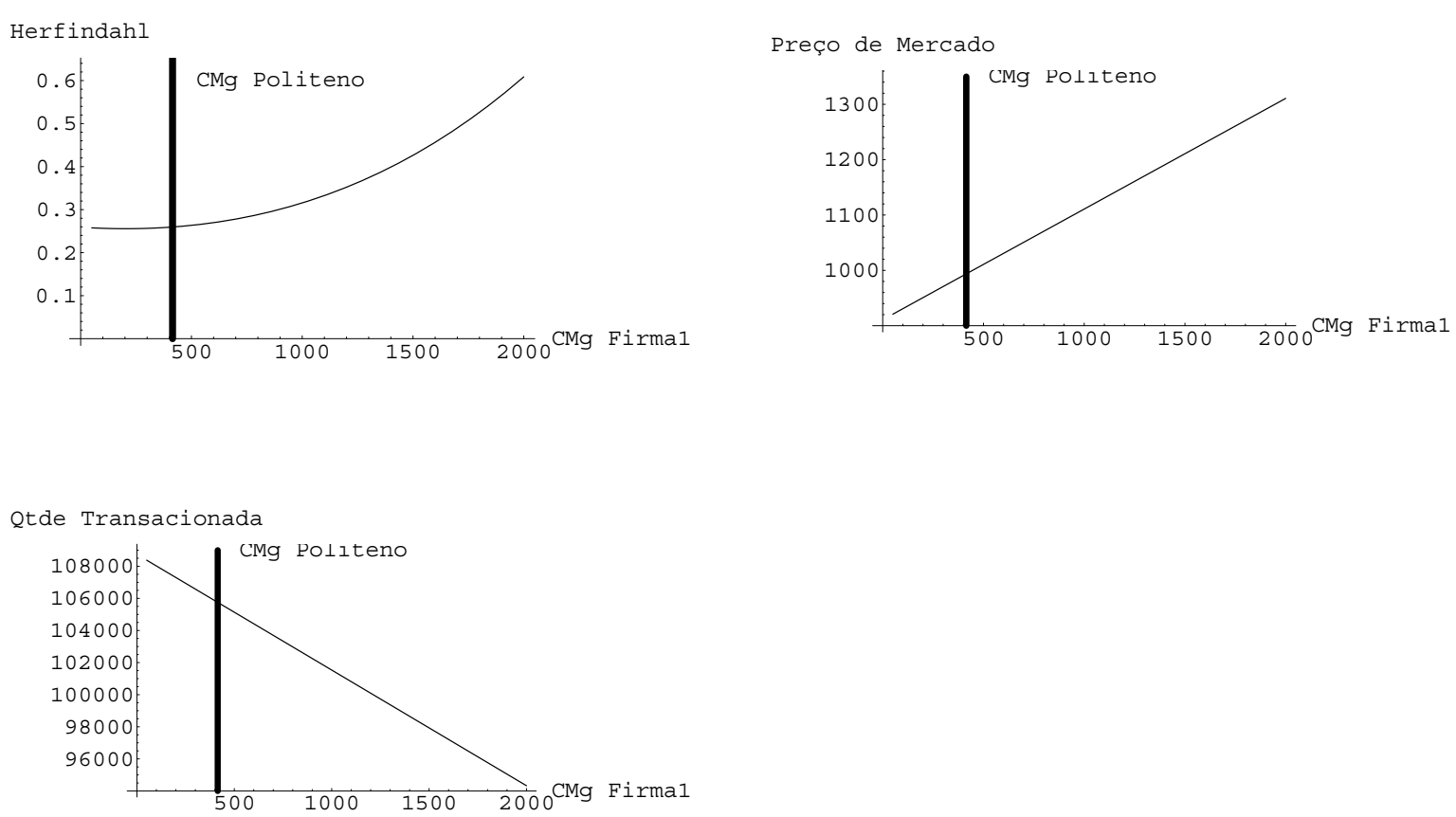

Fonte: Elaboração dos Autores.

No setor como um todo, do mesmo modo, a elevação do preço de eteno acima dos valores presentes tende a (1) elevar a concentração - medida pelo Índice Herfindahl-Hirschman, (2) elevar o preço pago pelos consumidores e (3) reduzir a quantidade vendida no setor.

Uma vez que não existem "eficiências" típicas desta operação - sinergias ou realocação eficiente da produção -, o aumento da concentração, mais o aumento do custo marginal dos rivais, leva a uma maior concentração e aumento de preços. Os ganhos em eficiência da fusão podem ocorrer apenas em termos dinâmicos (coordenação de novos investimentos), pois no quadro atual da indústria, a produção nos pólos já é integrada, regida por contratos de longo prazo, e não pode ser melhorada apenas pela mudança de controle acionário.

\subsection{Participações de mercado exógenas}

A segunda perspectiva para abordar a indústria, a mais utilizada em análises antitruste, é a que considera como exógenas as participações de mercado, dadas pelas capacidades produtivas e pela taxa de ocupação, e considera os efeitos sobre o mercado a partir da distribuição das participações das firmas. Assim, a partir dos dados de custos marginais calculados, e das participações de mercado obtidas a partir das capacidades de produção dos diferentes grupos, serão analisadas - por meio de três cenários - como a operação em tela irá afetar as principais variáveis relevantes do mercado de polietileno.

Inicialmente, caracteriza-se a situação em um cenário denominado "base", no qual a situação é análoga à situação anterior à fusão. A seguir são construídos alguns cenários para analisar os efeitos sobre os preços e sobre a concentração de mercado, com as seguintes características: 
- Cenário 1: Incorporação da empresa POLIALDEN ao Grupo Odebrecht - que veio a originar a empresa BRASKEM - sem alterações no preço do eteno;

- Cenário 2: Elevação no preço do eteno vendido pela empresa COPENE de 10,77\% sem a incorporação da empresa POLIALDEN ao Grupo Odebrecht;

- Cenário 3: Incorporação da empresa POLIALDEN ao Grupo Odebrecht com aumento de $10,77 \%$ no preço de eteno vendido pela empresa COPENE.

Estes cenários foram escolhidos levando em conta a concentração horizontal e a possibilidade de manipulação estratégica do preço de eteno. A Tabela 1 mostra a capacidade instalada de produção de eteno em 2001, conforme dados da ABIQUIM. A empresa BRASKEM controla, após a fusão, $82 \%$ da capacidade instalada de produção de eteno no Brasil, o que pode dar a ela o poder de definir preços relativos deste insumo dentro do País.

Tabela 1 - Capacidade instalada de produção de eteno - COPENE, COPESUL e PQU

\begin{tabular}{lccc}
\hline Empresa & Localização & Capacidade Instalada em 2001 (t/ano) & \% sobre o Total \\
\hline COPENE & BA & 1.200 .000 & $42 \%$ \\
COPESUL & RS & 1.135 .000 & $40 \%$ \\
Petroquímica União & SP & 500.000 & $18 \%$ \\
Total & & 2.835 .000 & $100 \%$ \\
\hline
\end{tabular}

Fonte: ABIQUIM (2002).

A análise atual simplifica dois fatos importantes por falta de informações adequadas sobre as empresas envolvidas: a) considera constante o efeito da produção da empresa localizada em Bahía Blanca na Argentina, ${ }^{20}$ que faz parte do Mercosul e b) não considera as plantas que entrarão em operação no pólo da empresa Rio Polímeros. ${ }^{21}$ No primeiro caso, considerando constante a produção da Argentina destinada ao mercado brasileiro, a inclusão destas plantas tem algum efeito sobre a magnitude do resultado da simulação, mas não do seu "sinal", ou seja, as conclusões seriam as mesmas, embora com menor intensidade. Quanto à entrada em operação do novo pólo do Rio de Janeiro, seu efeito será significativo, todavia será diluído no decorrer dos próximos 5 anos, devido a seus compromissos de exportação, de modo que o problema concorrencial deve se manifestar ainda por um tempo significativo.

Desta forma, a simulação que sintetiza estas questões é aquela expressa no Cenário 3, que incorpora, além da fusão horizontal, uma elevação no preço do principal insumo para a produção de polietilenos - o eteno - e mimetiza as recentes elevações observadas no preço de venda da empresa COPENE comparativamente aos preços praticados em outros pólos petroquímicos.

20 A empresa Dow Chemical inaugurou sua planta de polietilenos em dezembro de 2000, com a capacidade de produção de 200 mil toneladas/ano.

21 A empresa RIOPOL iniciou sua produção em 2005, com a capacidade de produção de 540 mil toneladas/ano, produzindo tanto PEAD quanto PEBDL. 
Figura 4 - Evolução do preço do eteno - COPENE/BRASKEM, PQU e COPESUL

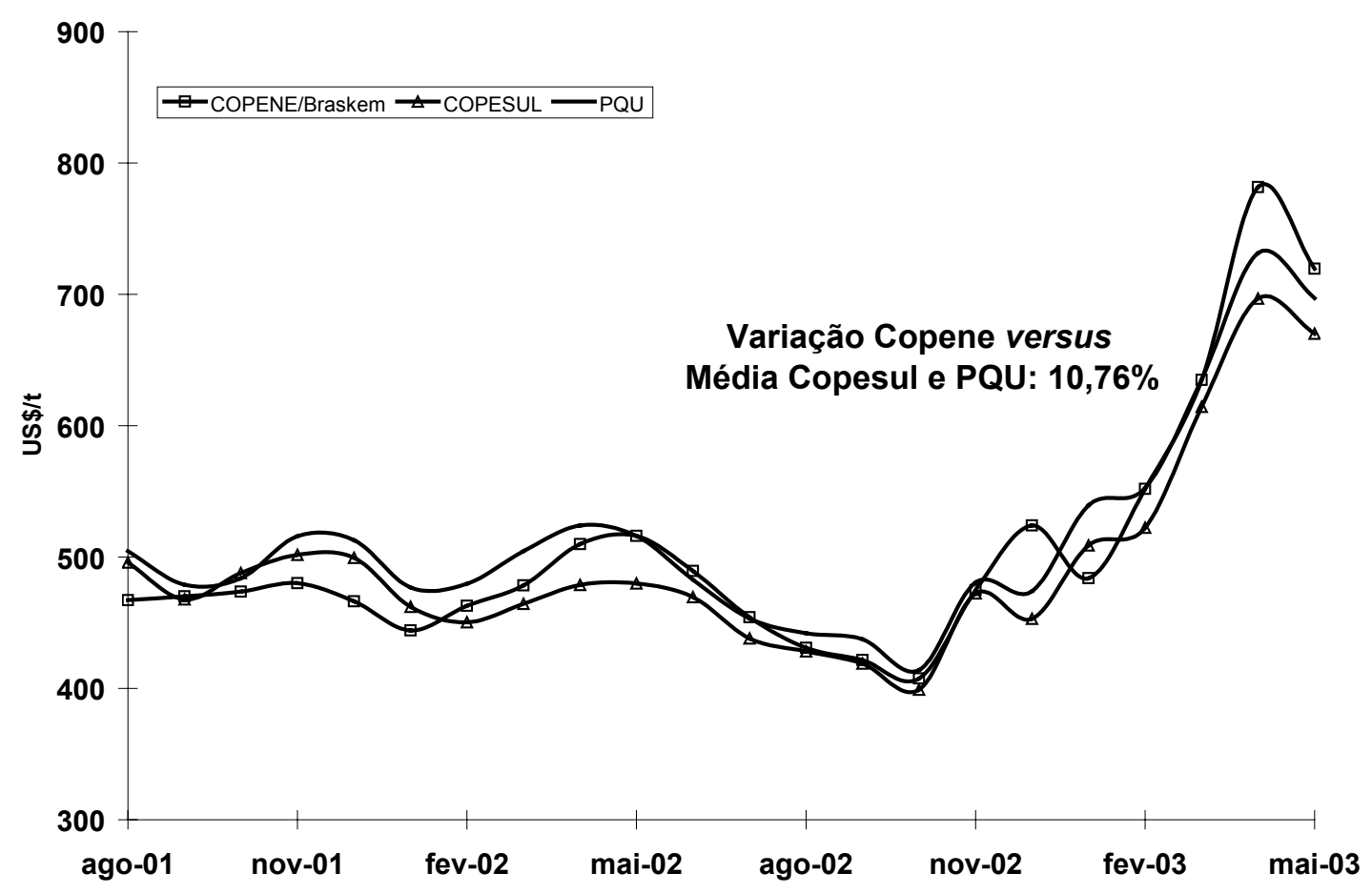

Fonte: Elaboração dos Autores.

A figura mostra como os preços de eteno no pólo da Bahia, que eram geralmente mais baixos do que na empresa COPESUL, passaram recentemente a apresentar valores mais elevados em vários períodos, antes mesmo da fusão envolvendo a formação da empresa BRASKEM ter sido aprovada pelo CADE (Conselho Administrativo de Defesa Econômica).

Com estes dados, foi escolhida a variação dos preços relativos de eteno nos três pólos petroquímicos observada em dezembro de 2002 para balizar a variação nos preços observada no Cenário 2. Cada um dos cenários utiliza os seguintes valores:

1. Custos Marginais de cada planta do setor;

2. Capacidades de cada planta do setor;

3. Elasticidades-Preço da Demanda por setor.

As elasticidades-preço da demanda por setor foram obtidas a partir da estimação mostrada no Anexo 2 para a especificação de demanda isoelástica. Os resultados ali expostos indicam que a demanda de mercado de polietileno é pouco elástica: cerca de 0,398 . Ou seja, uma elevação de $1 \%$ no preço de mercado faz com que a quantidade demandada, coeteris paribus, se reduza em 0,398\%. Serão realizadas estimações adicionais utilizando valores de 0,6 e 0,8 para esta variável. ${ }^{22}$

22 Com relação aos valores das elasticidades, a literatura apresenta uma ampla gama de valores. Por exemplo, Scarlett et al. (1997) apresentam uma elasticidade-preço para os produtos da família dos polietilenos - considerando somente a produção de embalagens - da ordem de 0,85 para os EUA. É de se esperar que o valor para a elasticidade-preço da demanda por polietileno seja significativamente inferior, uma vez que se está considerando mais produtos (ou seja, o número de substitutos possíveis é menor). De qualquer maneira, as simulações a seguir buscam incluir valores próximos a 0,86 para este parâmetro, a título de checagem de consistência dos resultados aqui apresentados. 
Tabela 2 - Estimativas de participação de mercado

\begin{tabular}{lc}
\hline Empresa & $\%$ de Mercado \\
\hline Braskem & $32,04 \%$ \\
Ipiranga & $22,56 \%$ \\
Politeno & $15,34 \%$ \\
Triunfo & $7,22 \%$ \\
Polialden & $6,77 \%$ \\
Dow & $6,50 \%$ \\
Polietilenos União & $5,87 \%$ \\
Solvay & $3,70 \%$ \\
Total Geral & $100 \%$ \\
\hline
\end{tabular}

Fonte: ABIQUIM (2002).

Os Custos Marginais para as diferentes plantas foram obtidos a partir dos valores calculados na seção 3 do presente artigo. Foram obtidos valores para o Custo Marginal das diferentes plantas do setor variando os preços de eteno por pólo petroquímico - Rio Grande do Sul, São Paulo e Bahia. As participações de mercado foram estimadas com base nas capacidades por linha de produtos, obtidas a partir da ABIQUIM (2002).

Tendo coletado todos os dados necessários, o passo seguinte consiste em calcular os preços e as quantidades relevantes do mercado. O primeiro passo foi calcular o Índice Herfindahl-Hirschmann de concentração de mercado, de acordo com a participação de cada um dos grandes grupos do setor. Este índice é calculado da seguinte forma:

$$
H H I=\sum_{I}\left(s_{i}\right)^{2}
$$

Em que si refere-se à participação de mercado da firma i. De posse deste valor, foi calculado um preço associado a cada um dos cenários anteriormente descritos. Este preço foi calculado a partir da seguinte equação:

$$
\frac{P-\overline{C M g}}{P}=\frac{H H I}{\varepsilon_{D}}
$$

Em que P é o preço do produto no mercado final; HHI o índice de concentração; $\overline{C M g}$ é o custo marginal médio do setor, ponderado pelas respectivas capacidades de produção e $\varepsilon_{D}$ a elasticidade-preço da demanda do produto final.

Ainda que esta abordagem seja em grande medida distinta da anterior, as duas possuem a mesma base teórica, tendo em vista que esta equação também se baseia em um modelo de competição imperfeita Cournot. ${ }^{23}$ Os valores calculados estão expostos na Tabela 3.

23 Para mais detalhes, ver Tirole (1989). 
Tabela 3 - Resultados - cenários

\begin{tabular}{|c|c|c|c|c|c|c|}
\hline \multicolumn{7}{|l|}{ Participação Exógena de Mercado } \\
\hline \multirow{3}{*}{ Caso 1: Sem aumento do Preço Eteno } & \multicolumn{6}{|c|}{ Elasticidades-Preço da Demanda } \\
\hline & \multicolumn{2}{|c|}{0,399} & \multicolumn{2}{|c|}{0,6} & \multicolumn{2}{|c|}{0,8} \\
\hline & Pré Fusão & Pós Fusão & Pré Fusão & Pós Fusão & Pré Fusão & Pós Fusão \\
\hline $\mathrm{HHI}$ & 0,196 & 0,237 & 0,196 & 0,237 & 0,196 & 0,237 \\
\hline Preço & 828,15 & 1036,24 & 625,85 & 696,52 & 558,19 & 599,05 \\
\hline Share Braskem & $32,04 \%$ & $38,25 \%$ & $32,04 \%$ & $38,25 \%$ & $32,04 \%$ & $38,25 \%$ \\
\hline Custo Marginal (Média) & 421,48 & 421,93 & 421,48 & 421,93 & 421,48 & 421,93 \\
\hline \multirow{3}{*}{ Caso 2: Com aumento do Preço Eteno } & \multicolumn{6}{|c|}{ Elasticidades-Preço da Demanda } \\
\hline & \multicolumn{2}{|c|}{0,399} & \multicolumn{2}{|c|}{0,6} & \multicolumn{2}{|c|}{0,8} \\
\hline & Pré Fusão & Pós Fusão & Pré Fusão & Pós Fusão & Pré Fusão & Pós Fusão \\
\hline $\mathrm{HHI}$ & 0,196 & 0,237 & 0,196 & 0,237 & 0,196 & 0,237 \\
\hline Preço & 847,40 & 1055,80 & 640,40 & 709,66 & 571,16 & 610,36 \\
\hline Share Braskem & $32,04 \%$ & $38,25 \%$ & $32,04 \%$ & $38,25 \%$ & $32,04 \%$ & $38,25 \%$ \\
\hline Custo Marginal (Média) & 431,27 & 429,89 & 431,27 & 429,89 & 431,27 & 429,89 \\
\hline
\end{tabular}

Fonte: Elaboração dos Autores.

De acordo com os dados resultantes da simulação, o controle da empresa POLIALDEN pela empresa BRASKEM resultaria em um aumento do índice de concentração Herfindahl-Hirschmann em cerca de $20,74 \%$. Agregando ainda um aumento de preço de eteno de $10,76 \%$, a fusão - considerando os três cenários de elasticidade da demanda, e realizando uma média deles - provoca um aumento de preços de aproximadamente $14,5 \%$ em relação ao preço do cenário-base. ${ }^{24}$

Este modelo pode superestimar os efeitos da concentração sobre o aumento do preço médio do polietileno no mercado brasileiro, ao não considerar o teto de preços colocado pelas importações, que deve ser analisado no tópico a seguir, quando se discute a definição de mercado relevante. Todavia, o diferencial observado entre preços domésticos e importados dos polietilenos, embora talvez não permita um aumento de $14,5 \%$, como se calculou, permite um "pequeno mas não transitório aumento de preços", que é condição suficiente para afirmar a existência de perda de bem-estar econômico.

\section{Conclusão}

O objetivo do presente estudo foi realizar uma simulação dos efeitos da operação que deu origem à empresa BRASKEM. A simulação indicou aumento do poder de mercado desta empresa na produção de polietilenos, seja porque houve concentração na produção de polietilenos com a incorporação da empresa POLIALDEN ao grupo BRASKEM, seja pela capacidade da entidade resultante, a empresa BRASKEM, que participa simultaneamente do controle da COPENE e da

24 Pode-se observar, a partir da análise dos dados das duas tabelas anteriores, que a simulação que tende a mostrar resultados mais próximos, em termos de preços, aos efetivamente observados, é o de 0,399. 
COPESUL, de manipular preços e quantidades de eteno ofertadas aos concorrentes brasileiros da empresa BRASKEM na segunda geração.

Para avaliar os efeitos da operação foi realizada uma análise que simulou o comportamento das empresas produtoras de polietilenos e eteno em termos de fixação de preços e quantidades ofertadas. Quanto ao eteno, vale apenas relembrar que as plantas de polietileno situadas em um pólo dependem da produção cativa da planta de primeira geração do mesmo pólo, tendo em vista os custos de transporte do eteno. No mercado de polietilenos, a estrutura de mercado de oligopólio Cournot foi utilizada para simular o setor, por ser considerada a mais adequada para setores caracterizados por altas barreiras à entrada, produtos homogêneos e capacidade produtiva fixa por período de tempo significativo, como se observa na indústria química.

A análise antitruste, para representar efeitos de uma fusão, tem recorrido crescentemente a modelos de "calibração" do setor, para servir como base para comparar situações de equilíbrio de mercado, pré e pós-fusão. A fusão, quando é analisada pelas autoridades antitruste, ainda não apresentou, plenamente, os seus efeitos sobre o mercado - pois as participantes da fusão devem, racionalmente, manter um comportamento mais moderado do que aquele que as empresas seriam capazes, para aumentar a probabilidade de aprovação da operação - daí a utilidade destes modelos.

Foram realizados dois diferentes conjuntos de simulações. No primeiro deles, as participações de mercado das firmas decorrem dos seus diferentes custos marginais, portanto, as participações de mercado são endógenas, ou seja, resultantes do próprio modelo, dados os custos marginais assimétricos. As empresas com menores custos marginais alcançam maiores participações de mercado. Neste modelo, a manipulação de preços de eteno, principal componente do custo marginal, ocasiona a mudança de participações de mercado e, conseqüentemente, do grau de concentração e de preços e quantidades de equilíbrio no mercado de polietilenos.

O segundo conjunto de simulações considera as participações de mercado das diferentes firmas como dadas (exógenas), decorrentes das diferentes capacidades produtivas das firmas. A manipulação dos preços de eteno para cada planta e a mudança das participações de mercado (dadas exogenamente pela fusão) e de grau de concentração ocasionam, neste caso, a mudança de preços e quantidades de equilíbrio no mercado de polietilenos.

As principais conclusões destas simulações, partindo da formação da empresa BRASKEM e conseqüente controle da maior parcela da produção de eteno no Brasil e do aumento da concentração da capacidade produtiva no setor de polietilenos, foram:

1. Elevação do preço médio dos polietilenos no mercado brasileiro;

2. Redução da quantidade total de polietilenos consumida;

3. Elevação da concentração no setor, medida pelo Índice Herfindahl-Hirschmann;

4. Maior participação de mercado da empresa BRASKEM, o que reforça sua posição dominante.

Os fundamentos de teoria econômica que sustentam estes resultados são de que uma fusão que provoca aumento de concentração sem gerar sinergias significativas, que poderiam reduzir custos marginais, em um oligopólio do tipo Cournot, gera maior concentração, portanto, maiores margens de lucro e maiores preços aos consumidores.

Ademais, a concentração vertical permite a mudança de preços relativos de eteno em benefício das plantas do grupo que detém as centrais de matérias-primas, o que lhe permite aumentar o 
preço deste insumo para os rivais. Deste modo, pode aumentar os custos marginais dos rivais, ampliando sua própria participação no mercado de polietilenos e suas margens de lucro. Para capturar este efeito no modelo foram utilizados os preços de eteno efetivamente praticados nos diferentes pólos, e constatou-se que após a fusão haver se concretizado, o preço de eteno da empresa COPENE passou a ser, de maneira mais freqüente, mais elevado do que o da empresa COPESUL, sem qualquer justificativa de custos para tal. Assim, fez-se uma análise comparativa com um aumento conservador de preços de eteno da empresa COPENE, da ordem de $10 \%$.

Estes dois efeitos são suficientes para justificar um "pequeno, mas não transitório" aumento de preços para os consumidores brasileiros de polietilenos como resultado da formação da empresa BRASKEM. Embora os efeitos da formação da empresa BRASKEM sejam diluídos ao longo do tempo, existe, com certeza, a necessidade de controlar seus impactos anticoncorrenciais nos próximos anos.

\section{REFERÊNCIAS BIBLIOGRÁFICAS}

ABIQUIM. Anuário da indústria química brasileira. Associação Brasileira da Indústria Química, São Paulo, 2002. Paulo, 2003.

Estrutura da indústria química brasileira. Associação Brasileira da Indústria Química, São 2005.

. Anuário da indústria química brasileira. Associação Brasileira da Indústria Química, São Paulo,

Baker, J. Developments in antitrust economics. Journal of Economic Perspectives, v. 13, n. 1, p. 181-194, 1999.

Battese, G. E.; Coelli, T. J. Frontier production functions, technical efficiency and panel data: with application to paddy farmers in India. Journal of Productivity Analysis, 3, p. 153-169, 1992.

Compte, O.; Jenny, F.; Rey, P. Capacity constraints, mergers and collusion. 1996. Mimeografado.

Farrell, J.; Shapiro, C. Horizontal mergers: an equilibrium analysis. American Economic Review, v. 80, n. 1, p. 107-126, March 1990.

. Scale economies and synergies in horizontal merger analysis. Antitrust Law Journal, 2001.

Greene, W. H. Econometric analysis. $3^{\text {rd }}$ Edition. Prentice-Hall, 1996.

Kadiyali, V. Entry, its deterrence and accommodation: a study of the U.S. photographic film industry. The RAND Journal of Economics, v. 29, n. 3, 1996.

Kreps, D.; Scheinkman, J. Quantity precommitment and Bertrand competition yields Cournot outcomes. Bell Journal of Economics, 14, p. 326-337, 1983.

Lieberman, M. B. Excess capacity as a barrier to entry: an empirical appraisal. Journal of Industrial Economics, v. 35, n. 4, p. 607-625, 1987.

Maggi, G. Strategic trade policies with endogenous mode of competition. American Economic Review, v. 86, n. 1, p. 237-258, 1996.

Ordover, J. A.; Saloner, G.; Salop, S. C. Equilibrium vertical foreclosure. American Economic Review, v. 80, n. 1, p. 127-42, 1990.

Riordan, M. H.; Salop, S. C. Evaluating vertical mergers: a post Chicago approach. Antitrust Law Journal, 63, p. 513-568, 1995. 
Roberts, J.; Samuelson, L. An empirical analysis of dynamic, nonprice competition in an oligopolistic industry. Rand Journal of Economics, v. 19, n. 2, 1988.

Salop, S. C.; Scheffman, D. T. Raising rivals' costs. American Economic Review, (Papers and Proceedings), v. 73, n. 2, p. 267-71, 1983.

. Cost-raising strategies. Journal of Industrial Economics, v. 36, n. 1, p. 19-34, 1987.

Santana, L. M. de; Hasenclever, L.; Mello, J. M. C. de. Capacitação tecnológica e competitividade na petroquímica brasileira nos anos 1990: o caso de Camaçari-BA. Revista Brasileira de Inovação, v. 2, n. 1, p. 147-177, 2003.

Scarlett, L.; McCann, R.; Anex, R.; Volokh, A. Packaging, recycling and solid waste. California: Reason Foundation, 1997.

Scherer, F. M.; Ross, D. Industrial market structure and economic performance. Boston: Houghton-Mifflin, 1990.

Schmalensee, R. Horizontal merger policy: problems and changes. Journal of Economic Perspectives, v. 1, n. 2, p. 41-54, 1987.

SEAE (Secretaria de Acompanhamento Econômico do Ministério da Fazenda). Parecer COINP/COGPI/ SEAE/MF n $n^{\circ} 153$ de 5 de julho de 2002.

SEAE (Secretaria de Acompanhamento Econômico do Ministério da Fazenda) e SDE (Secretaria de Direito Econômico do Ministério da Justiça). Guia de Concentrações Horizontais. Portaria Conjunta $N^{\circ} 50$, de $1^{\circ}$ de Agosto de 2001.

Spector, D. Horizontal mergers, entry and efficiency defences. CEPREMAP-CNRS, $\mathrm{n}^{\circ}$ 06, 2002. Mimeografado.

Tirole, J. The theory of industrial organization. Cambridge (Mass.): MIT Press, 1989.

Viscusi, W. K.; Vernon, J. M.; Harrington Jr., J. E. Economics of regulation and antitrust. Cambridge (Mass.): MIT Press, 2000.

\section{APÊNDICES}

\section{APÊNDICE 1 - RESULTADOS DA ESTIMAÇÃO POR SEGMENTO}

$$
\begin{aligned}
& \mathrm{Q}_{\text {PEADt }}=\beta_{0}+\beta_{1} \mathrm{P}_{\text {PEADt }}+\beta_{2} \mathrm{Q}_{\text {PERFt }}+\beta_{3} \mathrm{Q}_{\mathrm{CDIt}}+\beta_{4} \mathrm{D} 1_{\mathrm{t}}+\varepsilon_{\mathrm{t}} \\
& \mathrm{Q}_{\text {PEBDLt }}=\beta_{5}+\beta_{6} \mathrm{P}_{\text {PEBDLt }}+\beta_{7} \mathrm{Q}_{\mathrm{CDI}}+\varepsilon_{\mathrm{t}} \\
& \mathrm{Q}_{\text {PEAD }}+\mathrm{Q}_{\text {PEBDL }}=\gamma_{1}+\gamma_{2} \mathrm{~L}+\gamma_{3} \mathrm{P}_{\text {ETENO }}-\beta_{1} \mathrm{P}_{\text {PEAD }}-\beta_{6} \mathrm{P}_{\text {PEBDL }}+\varepsilon_{\mathrm{t}}
\end{aligned}
$$


Tabela 4 - Resultados da estimação

\begin{tabular}{|c|c|c|c|}
\hline & \multicolumn{3}{|c|}{ Modelos } \\
\hline & 6 & 7 & 8 \\
\hline \multirow[t]{2}{*}{ Constante } & 33775,24 & 41137,69 & 61755,73 \\
\hline & $(2,354)$ & $(7,836)$ & $(5,572)$ \\
\hline \multirow[t]{2}{*}{$P_{P E A D}$} & $-12,065$ & & $-12,065$ \\
\hline & $(-2,019)$ & & $(-2,019)$ \\
\hline \multirow[t]{2}{*}{$\mathrm{P}_{\mathrm{PEBDL}}$} & & $-12,260$ & $-12,260$ \\
\hline & & $(-2,541)$ & $(-2,541)$ \\
\hline \multirow[t]{2}{*}{$Q_{\text {PERF }}$} & 365,878 & & \\
\hline & $(4,706)$ & & \\
\hline \multirow[t]{2}{*}{$Q_{C D I}$} & & $-5907,032$ & \\
\hline & & $(-4,138)$ & \\
\hline \multirow[t]{2}{*}{ D1 } & 6028,855 & & \\
\hline & $(2,066)$ & & \\
\hline \multirow[t]{2}{*}{$\mathrm{L}$} & & & $-15,065$ \\
\hline & & & $(-2,796)$ \\
\hline \multirow[t]{2}{*}{$P_{\text {ETENo }}$} & & & 30,664 \\
\hline & & & $(4,290)$ \\
\hline $\mathrm{R}^{2}$ Ajustado & 0,4066 & 0,2160 & 0,4988 \\
\hline
\end{tabular}

Obs: Estatísticas t entre parênteses.

APÊNDICE 2 - RESUlTAdOS DA ESTIMAÇÃO - SETOR AGREGADO

$$
\begin{aligned}
& \mathrm{Q}_{\mathrm{Tt}}=\beta_{0}+\beta_{1} \mathrm{P}_{\mathrm{POLt}}+\beta_{2} \mathrm{Q}_{\mathrm{INDt}}+\varepsilon_{\mathrm{t}} \\
& \mathrm{Q}_{\mathrm{Tt}}=\beta_{3}+\beta_{4} \mathrm{~L}_{\mathrm{t}}+\beta_{5} \mathrm{P}_{\mathrm{ETENOt}}+\beta_{6} \mathrm{P}_{\mathrm{POLt}}+\varepsilon_{\mathrm{t}}
\end{aligned}
$$

Tabela 5 - Resultados da estimação

\begin{tabular}{lrr}
\hline & \multicolumn{1}{c}{ Modelos } \\
\cline { 2 - 3 } & \multicolumn{1}{c}{9} & \multicolumn{1}{c}{10} \\
\hline Constante & $111.741,7$ & 125.366 \\
& $(3,672)$ & $(8,908)$ \\
$\mathrm{P}_{\mathrm{POL}}$ & $-43,948$ & 17,325 \\
& $(-2,415)$ & \\
$\mathrm{Q}_{\text {IND }}$ & 503,365 & \\
& $(2,707)$ & $-5,998$ \\
$\mathrm{~L}$ & & $(-2,460)$ \\
& & 15,578 \\
$\mathrm{P}_{\text {ETENO }}$ & & $(3,364)$ \\
\hline R $^{2}$ Ajustado & & 0,350 \\
\hline
\end{tabular}

Obs: Estatísticas t entre parênteses. 


\section{APÊNDICE 3 - RESULTADOS DA ESTIMAÇÃO - SETOR (ESPECIFICAÇÃO ISOELÁSTICA)}

$$
\begin{aligned}
& \ln \left(\mathrm{Q}_{\mathrm{Tt}}\right)=\beta_{0}+\beta_{1} \ln \left(\mathrm{P}_{\mathrm{POLt}}\right)+\beta_{2} \ln \left(\mathrm{Q}_{\mathrm{CDIt}}\right)+\varepsilon_{\mathrm{t}} \\
& \ln \left(\mathrm{Q}_{\mathrm{Tt}}\right)=\beta_{3}+\beta_{4} \ln \left(\mathrm{L}_{\mathrm{t}}\right)+\beta_{5} \ln \left(\mathrm{P}_{\mathrm{ETENOt}}\right)+\beta_{6} \ln \left(\mathrm{P}_{\mathrm{POLt}}\right)+\varepsilon_{\mathrm{t}}
\end{aligned}
$$

Tabela 6 - Resultados da estimação

\begin{tabular}{lrr}
\hline & \multicolumn{2}{c}{ Modelos } \\
\cline { 2 - 3 } & \multicolumn{1}{c}{11} & \multicolumn{1}{c}{12} \\
\hline Constante & 14,648 & 11,263 \\
& $(20,887)$ & $(16,647)$ \\
$\ln \left(P_{\text {POL }}\right)$ & $-0,389$ & 0,131 \\
& $(-3,865)$ & $(1,272)$ \\
$\ln \left(Q_{\text {CDI }}\right)$ & $-0,297$ & \\
& $(-5,685)$ & $-0,148$ \\
$\operatorname{Ln}(L)$ & & $(-2,555)$ \\
& & 0,125 \\
$\ln \left(P_{\text {ETENO }}\right)$ & & $(3,908)$ \\
\hline$R^{2}$ Ajustado & & 0,382 \\
\hline
\end{tabular}

Obs: Estatísticas t entre parênteses.

\section{APÊNDICE 4 - ESTIMATIVA DE FRONTEIRA ESTOCÁSTICA}

$$
\ln \left(\mathrm{CT}_{\mathrm{it}}\right)=\beta_{0}+\beta_{1} \ln \left(\mathrm{VA}_{\mathrm{it}}\right)+\varepsilon_{\mathrm{it}}-\mathrm{v}_{\mathrm{it}}
$$

Tabela 7 - Resultados do modelo de fronteira estocástica

\begin{tabular}{lr}
\hline & \multicolumn{1}{c}{1} \\
\hline Constante & 0,924 \\
$\ln \left(\mathrm{VA}_{\mathrm{it}}\right)$ & $(0,844)$ \\
$\sigma^{2}$ & 0,657 \\
& $(6,106)$ \\
$\mu$ & 0,482 \\
$\gamma$ & $(2,294)$ \\
& 1,122 \\
$\eta$ & $(3,018)$ \\
& 0,653 \\
Number of Observations & $(6,690)$ \\
Log-Likelihood & 0,027 \\
LR Test of One-sided Error (d.f.) & $(2,765)$ \\
\hline
\end{tabular}

Obs: Estatísticas t entre parênteses. 